\title{
Garcin syndrome as the initial presentation of metastatic adenocarcinoma of the prostate
}

\author{
Hiroki Kubota $\cdot$ Hiromichi Naruyama $\cdot$ Masahito Hirose $\cdot$ \\ Katsuhiro Fukuta · Yasue Kubota • Yasuyuki Yamada • \\ Kenjiro Kohri
}

Received: 30 November 2011 / Accepted: 1 February 2012/Published online: 17 March 2012

(C) The Japan Society of Clinical Oncology 2012

\begin{abstract}
We describe an unusual case of metastasis of the skull base from prostate cancer. A 64-year-old man was referred for progressive cranial nerve palsies. Brain magnetic resonance imaging showed a metastatic tumor in the right petrous bone. He was presenting Garcin syndrome indicating involvement of the right III, IV, V, VI, VII, VIII, IX, X, and XII cranial nerves. The serum PSA level was $5118 \mathrm{ng} / \mathrm{mL}$ (standard threshold $4 \mathrm{ng} / \mathrm{mL}$ ). A transrectal needle biopsy was performed, and poorly differentiated adenocarcinoma was detected in the prostate. Therefore, he was finally diagnosed with skull base metastasis of the prostate cancer. Androgen deprivation therapy and external beam radiation therapy to the skull base resulted in complete resolution of the cranial nerve palsies for more than 30 months. This is the first reported case of metastatic prostate cancer presenting as Garcin syndrome.
\end{abstract}

Keywords Garcin syndrome - Prostate cancer - Skull base metastasis

\section{Introduction}

The high incidence of prostate cancer in men over the age of 50 years is well recognized [1]. Clinical symptoms of

\footnotetext{
H. Kubota $(\bowtie) \cdot$ H. Naruyama · M. Hirose $\cdot$ K. Fukuta

Y. Yamada

Department of Urology, Kainan Hospital, 396 Minamihonden,

Maegasu-cho, Yatomi, Aichi 498-8502, Japan

e-mail: urokubo@gmail.com

Y. Kubota $\cdot$ K. Kohri

Department of Nephro-urology, Nagoya City University

Graduate School of Medical Science, 1 Kawasumi, Mizuho-cho,

Mizuho-ku, Nagoya, Aichi 467-8601, Japan
}

prostate cancer are normally inconspicuous, excluding disturbance of urination, macrohematuria, and bone pain. Cranial nerve palsies, which are usually caused by intracranial metastasis of the carcinoma, are very uncommon in prostate cancer.

Garcin syndrome is characterized by progressive involvement of the cranial nerves culminating in the total unilateral paralysis of all or most cranial nerves, with an absence of motor or sensory signs in the limbs and without any signs of increased intracranial pressure [2]. This unusual syndrome is normally associated with primary nasopharyngeal tumor and a variety of metastatic tumors that invade the skull base.

This report describes a rare case of skull base metastasis from prostate cancer presenting as Garcin syndrome. As far as we know, this is the first reported case of Garcin syndrome caused by prostate cancer.

\section{Case report}

The patient was a 64-year-old man who had been treated by an ophthalmologist since April 2006 due to diplopia. Because of gradual progression of right facial palsy, dysarthria, voice changes, and dysphagia, he was referred to the Department of Neurosurgery of our hospital on 26 September 2006. Neurological examination revealed right multiple cranial nerve palsies. Right ptosis was observed and right eye movement was restricted. The ciliary sign was positive. Right facial sensation and movement were weakened, and there was deviation of the jaw to the right on opening the mouth. He had atrophy of the right half of the tongue and the tongue deviated to the right on protrusion. Husky voice and dysphagia were obvious. However, he did not show any motor or sensory signs in the limbs, 
and no signs of increase in intracranial pressure were recognized. He was finally diagnosed with Garcin syndrome indicating involvement of the right III, IV, V, VI, VII, VIII, IX, X, and XII cranial nerves.

Magnetic resonance imaging (MRI) of the head showed a tumor in the right petrous bone (Fig. 1). The signal intensity of the tumor was similar to that of gray matter of the brain on both T1- and T2-weighted imaging. The tumor was heterogeneously enhanced on gadolinium-enhanced T1-weighted imaging. A metastatic disease was suspected as the most likely cause, rather than a meningioma. Bone scintigraphy indicated multiple bone metastases in the skull bone, vertebrae, ribs, ilium, and femur.

Cerebrospinal fluid examination including cytology was intact, and a complete blood count and blood chemistry were all within normal ranges. The serum PSA level was $5118 \mathrm{ng} / \mathrm{mL}$ (standard threshold $4 \mathrm{ng} / \mathrm{mL}$ ). On digital rectal examination, the bilateral lobes of the prostate were enlarged and the surface was uneven with a stony consistency. A transrectal needle biopsy was performed, and the patient was diagnosed with poorly differentiated adenocarcinoma of the prostate (Fig. 2). Gleason score was $5+4=9$. MRI of the pelvis revealed that the tumor had extended to a seminal vesicle and the bladder (Fig. 3).

Androgen deprivation therapy was immediately commenced, and $3000 \mathrm{cGy}$ of external beam radiation therapy was administered to the skull base. The 3-month follow-up head MRI showed significant regression in the skull base mass (Fig. 4). The cranial nerve palsies had improved greatly. The serum PSA level was $8.1 \mathrm{ng} / \mathrm{mL}$ at the 6-month follow-up. However, the carcinoma of the prostate

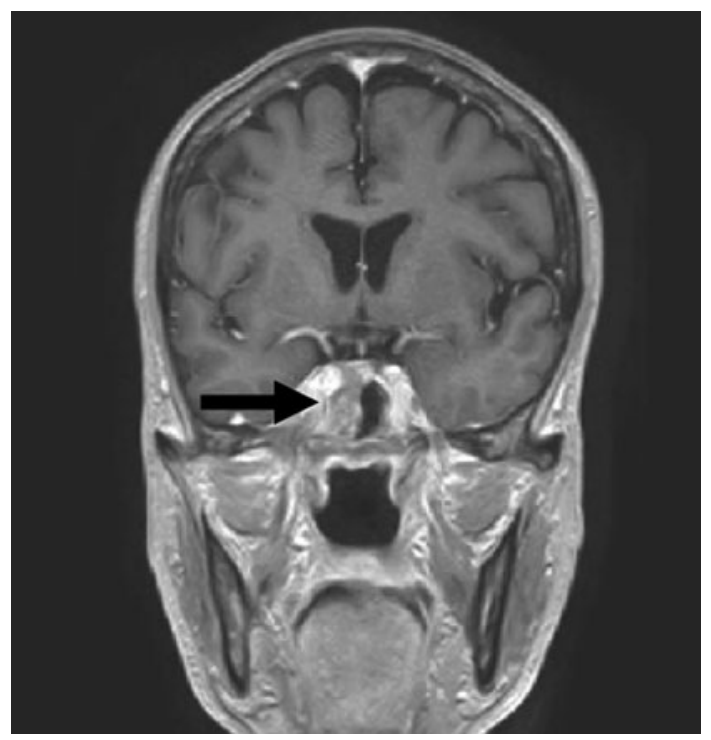

Fig. 1 Gadolinium-enhanced T1-weighted imaging of the coronal plane, demonstrating a metastatic tumor at the right petrous bone (arrow) subsequently became castration-refractory. Thus, we performed chemotherapy with docetaxel and the serum PSA level decreased by more than $50 \%$ for a while. In September 2009, right facial palsy gradually progressed, and diplopia recurred. Furthermore, multiple brain metastases appeared. The patient was re-admitted and died of systemic cancer progression on 15 November 2009.

\section{Discussion}

Prostate cancer commonly metastasizes to lymph nodes and bones. Vertebral bodies, pelvic bones, femur, and ribs are frequently involved in skeletal metastasis. The skull

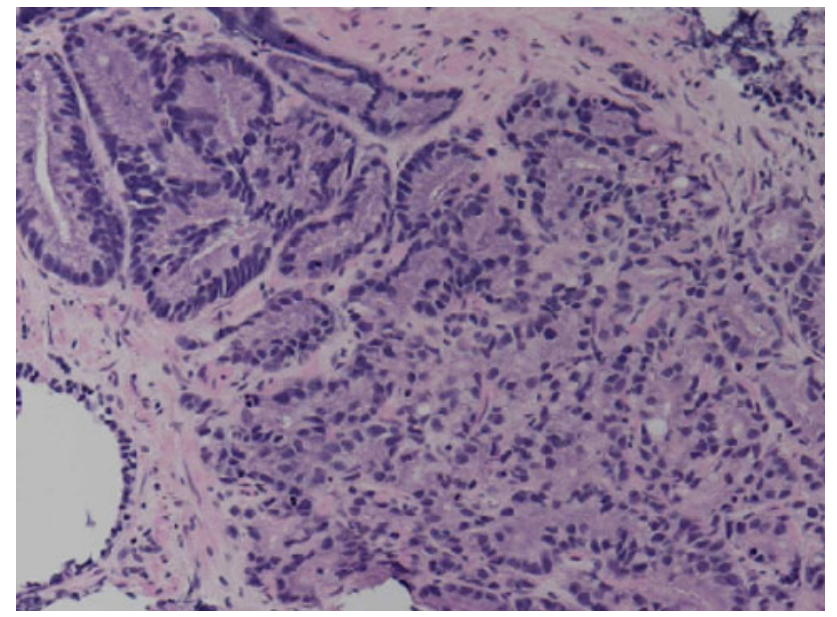

Fig. 2 Histopathology of a prostate needle biopsy showing poorly differentiated adenocarcinoma. H\&E $\times 100$

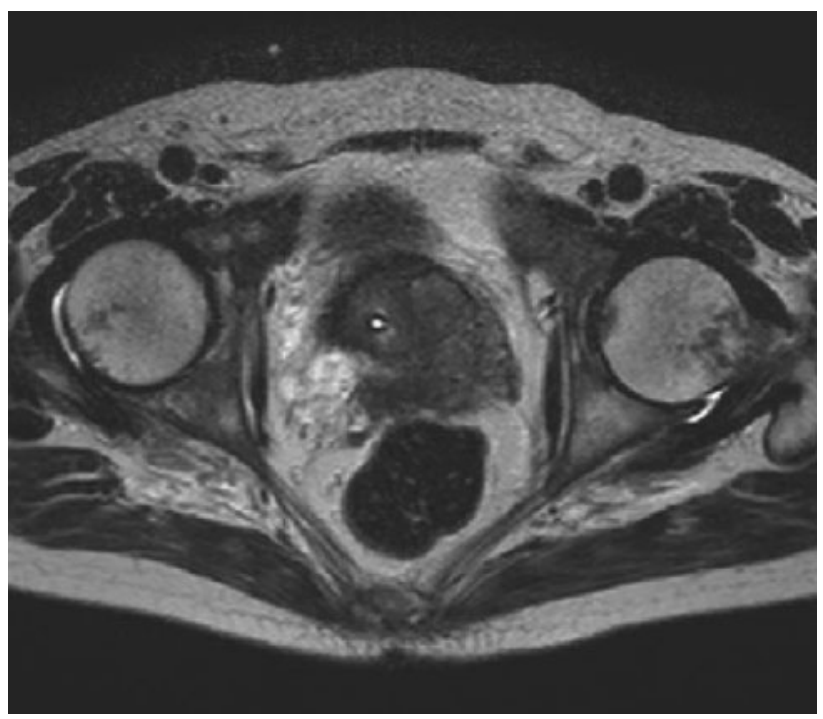

Fig. 3 T2-weighted imaging of the axial plane, showing prostate cancer that had extended to the extracapsular lesion and a seminal vesicle 


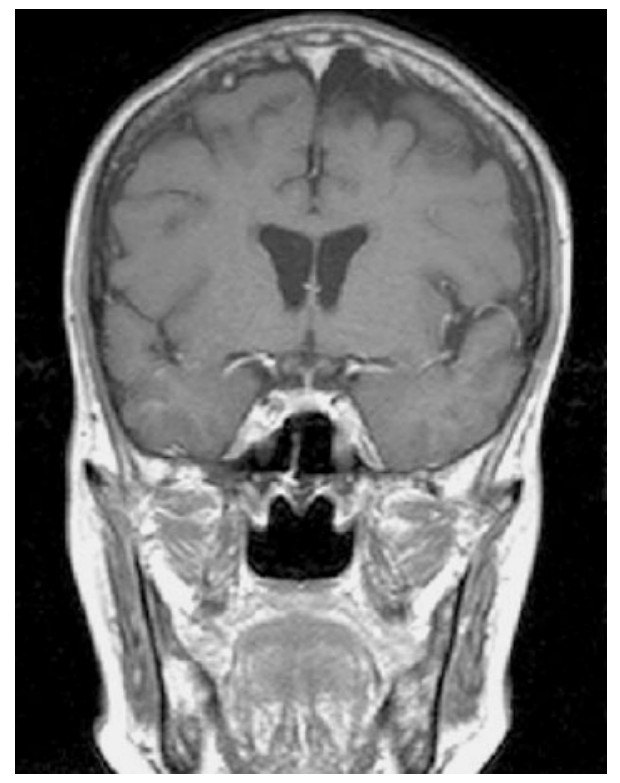

Fig. 4 Approximately 6 months after the EBRT, the metastatic tumor had almost disappeared

base is considered an unusual site for bone metastasis. A retrospective study revealed that the incidence of metastasis of prostate cancer to the skull base was $2 \%$ [3]. However, the gradual progression of tumors located in such a lesion may result in cranial nerve deficits and have a large impact on the patient's quality of life [4]. Such patients usually suffer from a variety of symptoms, because many different cranial nerves can be involved, whether alone or in combination [5].

A number of reports regarding cranial nerve deficits caused by skull base metastasis from prostate cancer have been reported [3-10]. Long et al. [3] reported that cranial nerve deficits were detected in $18 \%$ of patients with skull base metastasis from prostate cancer. O'Sullivan et al. reported that the most common palsies were of the $\mathrm{V}$ (facial paresthesia 19\%), VI (diplopia 22\%), and XII (deviation of tongue 19\%) cranial nerves [9]. Greenberg et al. identified five clinical syndromes-the orbital, parasellar, middle-fossa, jugular foramen, and occipital condyle syndromes-from a study of 43 patients presenting skull base metastasis [5]. Extensive skull base erosion may cause Garcin syndrome, characterized by progressive ipsilateral paralysis of at least seven cranial nerves [10]. However, there are no reports of Garcin syndrome due to metastatic prostate cancer of the skull base in the English literature.

Cranial neuropathy caused by skull base metastasis is usually seen in patients with advanced castration-refractory prostate cancer, and it occurs late in the disease process. Cranial neuropathy thus normally indicates a very poor prognosis, and the expected median survival time is
3-5 months [7-9]. Patients who have cranial nerve deficits as the initial presenting feature of prostate cancer are very rare. Such patients are considered to be able to survive longer, because they do not normally have castrationrefractory disease.

A case series indicated that EBRT to the skull base combined with corticosteroid was extremely effective at achieving palliative benefits [9]. Another study also revealed that EBRT to the whole brain or to the skull base were both successful [4]. The radiation dose schedule is controversial. However, $3000 \mathrm{cGy}$ in 10 fractions or 2000 cGy in 5 fractions are both regarded as standard palliative treatment regimens.

Here, we report for the first time a rare case of metastatic prostate cancer presenting as Garcin syndrome. Although intracranial metastasis from prostate cancer is less common, the present case indicates that metastatic prostate cancer should be considered in the differential diagnosis of males over the age of 50 years presenting with an atypical meningeal lesion involving the cranial nerves.

Conflict of interest The authors declare that they have no conflict of interest.

\section{References}

1. Jemal A, Siegel R, Ward E (2009) Cancer statistics, 2009. CA Cancer J Clin 59:225-249

2. Adams R, Victor M, Ropper AH (1997) Principles of neurology. In: The major categories of neurologic diseases. 6th edn. McGraw-Hill, New York, p 685

3. Long MA, Husband JE (1999) Features of unusual metastases from prostate cancer. Br J Radiol 72(862):933-941

4. McDermott RS, Anderson PR, Greenberg RE, Milestone BN, Hudes GR (2004) Cranial nerve deficits in patients with metastatic prostate carcinoma: clinical features and treatment outcomes. Cancer 101(7):1639-1643

5. Greenberg HS, Deck MD, Vikram B, Chu FC, Posner JB (1981) Metastasis to the base of the skull; clinical findings in 43 patients. Neurology 31(5):530-537

6. Seymore CH, Peeples WJ (1988) Cranial nerve involvement with carcinoma of prostate. Urology 31(3):211-213

7. Svare A, Fossa SD, Heier MS (1988) Cranial nerve dysfunction in metastatic cancer of the prostate. Br J Urol 61(5):441-444

8. Ransom DT, Dinapoli RP, Richardson RL (1990) Cranial nerve lesions due to base of the skull metastases in prostate carcinoma. Cancer 65(3):586-589

9. O'Sullivan JM, Norman AR, McNair H, Dearnaley DP (2004) Cranial nerve palsies in metastatic prostate cancer-results of base of skull radiotherapy. Radiother Oncol 70(1):87-90

10. Kolias AG, Derham C, Mankad K, Hasegawa H, O'Kane R, Ismail A, Phillips NI (2010) Multiple cranial neuropathy as the initial presentation of metastatic prostate adenocarcinoma: case report and review of literature. Acta Neurochir (Wien) 152(7): $1251-1255$ 